\title{
Dirac Equation in a 5-dimensional Kaluza-Klein Theory
}

\author{
Mustaqeem ANSARI ${ }^{1}$
}

\begin{abstract}
Dirac equation is discussed in 5-dimensional space time having topology $M^{4} \times T^{1}$, where $M^{4}$ and $T^{1}$ both are curved. It is shown that 4-dimensional fermion can be obtained from 5-dimensional fermion, as a result of compactification of extra dimension. It is found that the realistic 4-dimensional fermions are possible in higher modes earlier than those in lower modes during the course of expansion of 4-dimensional universe. 4-dimensional Dirac equation, obtained from 5-dimensional Dirac equation after compactification, is solved for an arbitrary modes for superheavy as well as light (realistic) fermions. Time-dependence of polarization vector and magnetization density, as a result of Gordon-decomposition of the current vector for 4-dimensional spin- $\frac{1}{2}$ field (with arbitrary mode), is exhibited.
\end{abstract}

Keywords: Fermions, Dirac equation, Kaluza-Klein theory, energy- momentum tensor.

PACS No: 11.10.K k, 03.65-w, 04.20-q.

\section{Introduction}

In the context of unification of gravity with gauge interactions, Kaluza-Klein type theories [1-4] are good candidates. In these theories, the spacetime is supposed to have the topology $M^{4} \times T^{n-4}$ (n is the total number of dimension), where $M^{4}$ denotes the usual paracompact four-dim. spacetime (flat or curved) and $T^{n-4}$ is the extra (n-4)-dim.compact manifold.The observed universe is 4-dimensional, hence it is supposed that the extramanifold due to gauge interactions might be compact and very small in size so that these are not observed today. Physically, it is very much appealing to think that $T^{n-4}$ manifold is curved due to its compact nature[5]. The action for higher-dim. gravity is $s=\int d^{n} x \sqrt{-g} R$ where $g=\operatorname{det} g_{\mu \nu} \quad\left(g_{\mu \nu}\right.$ is the metric tensor for the n-dimensional space time) and $\mathrm{R}$ is the Ricci curvature scalar for $M^{4} \times T^{n-4}$. n-dimensional Einstein's equations derived from the above action yields that $T^{n-4}$ can be curved (1) when $M^{4}$ is also curved or (2) action contains some lagrangian for matter field also so that energymomentum tensor is non-vanishing[6]. This discussion shows that if $M^{4}$ is curved, the

\footnotetext{
${ }^{1}$ Department of Mathematics, North-Eastern Hill University, NEHU Campus, Shillong-793022, India E-Mail: mansari@nehu.ac.in , mansari12_2006@yahoo.co.in
} 
extra-dim. manifold will definitely be curved. Motivated by this idea, the line-element for 5-dim. space time is taken as

$$
d s^{2}=d t^{2}-a^{2}(t)\left[d x_{1}^{2}+d x_{2}^{2}+d x_{3}^{2}\right]-A^{2}(t) d y^{2}
$$

Using the metric tensor from (1.1) in the Einstein's field equations[7] derived from the above action, it is interesting to see that

$$
a A=1
$$

Thus (1.1) deserves a model in which one spatial dimension shrinks with time while the other three expand. Earlier, Chodos and Detweiler[8] have considered this type of spacetime.

In 4-dim. spacetime, Dirac equation has been solved and discussed by many authors[9]. The aim of present paper is to discuss and solve Dirac equation for spin- $\frac{1}{2}$ field $\Psi_{5}$ in 5-dim. spacetime (1.1) for different time-function a(t).

The degrees of freedom for a spin- $\frac{1}{2}$ field $\Psi$ in $\mathrm{n}$ dimensions[10] is given by $2^{\alpha}$ where $\alpha=\frac{n}{2}$ (if $\mathrm{n}$ is even) and $\alpha=\frac{(n-1)}{2}$ (if $\mathrm{n}$ is odd). The dimension of the space-time, here, is 5, so degrees of freedom for $\Psi$ is 4 . The flat space Dirac matrices in 5-dim. will be $\tilde{\gamma}_{0}, \tilde{\gamma}_{1}, \tilde{\gamma}_{2}, \tilde{\gamma}_{3}, \tilde{\gamma}_{5}$ where $\tilde{\gamma}_{5}=\tilde{\gamma}_{0} \tilde{\gamma}_{1} \tilde{\gamma}_{2} \tilde{\gamma}_{3}$ (other $\gamma-$ matrices are the usual standard matrices for 4-dim.)[11]. Now for Weyl's transformation a matrix $\bar{\gamma}=\tilde{\gamma}_{0} \tilde{\gamma}_{1} \tilde{\gamma}_{2} \tilde{\gamma}_{3} \tilde{\gamma}_{5}=1$ can be defined. So operation of chiral projection operators $\frac{1}{2}(1 \pm \bar{\gamma})$ on the Dirac equation

$$
i \tilde{\gamma}^{\mu} \partial_{\mu} \Psi_{5}+m_{5} \Psi_{5}=0
$$

shows that chiral fermions are not possible in 5-dim.[10]. Hence $m_{5}$, the mass of $\Psi_{5}$ is not zero. This is true for fermions in curved spacetime also which obey the Dirac equation

$$
i \gamma^{\mu} D_{\mu} \Psi_{5}+m_{5} \Psi_{5}=0
$$

where $\gamma^{\mu}$ are curved space Dirac-matrices and $D_{\mu}$ denotes covariant derivatives in curved spacetime (1.1).

The paper is planned as follows: Section 2 contains 5-dim. Dirac Equation in the spacetime (1.1) and a brief discussion on the effective mass of 4-dim. fermion produced by 5-dim. fermion is given. Section 3 contains some explicit examples of solutions for 5-dim. 
Dirac Equation. In the last section, Gordon-decomposition[12] of 4-dim. $\Psi_{4}$, obtained after compactification of 5.dim spacetime, has been discussed.

$\hbar=c=1$ is used as fundamental unit where $\hbar$ and c carry their usual meaning. Here indices $a, \mu \cdots=0,1,2,3,5$.

\section{Dirac Equation for $\Psi_{5}$}

The vierbein $h_{a}^{\mu}$ on the manifold $M^{4} \times T^{1}\left(T^{1}\right.$ is circle) is defined as

$$
h_{a}^{\mu} h_{b}^{\nu} g_{\mu \nu}=\eta_{a b}
$$

where $(\mu, \nu)$ are curved space indices, $(\mathrm{a}, \mathrm{b})$ are flat space indices,$g_{\mu \nu}$ is the curved space metric tensor and $\eta_{a b}$ is the Minkowskian metric. So, in the space time (1.1)

$$
h_{0}^{0}=1, h_{1}^{1}=h_{2}^{2}=h_{3}^{3}=a^{-1}(t), h_{5}^{5}=A^{-1}(t)=a(t)
$$

The operator $D_{\mu}$ in (1.4) is defined as [13]

$$
D_{\mu}=\partial_{\mu}-\Gamma_{\mu}
$$

where $\Gamma_{\mu}$ is the spin coefficient or Ricci rotation coefficient given as

$$
\Gamma_{\mu}=\frac{1}{4}\left(\partial_{\mu} h_{a}^{\rho}+\left\{\begin{array}{c}
\rho \\
\sigma \mu
\end{array}\right\} h_{a}^{\sigma}\right) h_{b}^{\nu} g_{\rho \nu} \bar{\gamma}^{a} \bar{\gamma}^{b}
$$

where $\left\{\begin{array}{c}\rho \\ \sigma \mu\end{array}\right\}$ is the affine-connection.

$\tilde{\gamma}^{a}$ are flat space Dirac matrices satisfying the anti-commutation rule

$$
\left\{\tilde{\gamma}^{a}, \tilde{\gamma}^{b}\right\}=2 \eta^{a b}
$$

Curved space Dirac matrices $\gamma^{\mu}$ satisfy

$$
\left\{\gamma^{\mu}, \gamma^{\nu}\right\}=2 g^{\mu \nu}
$$

$\gamma^{\mu}$ is related to $\tilde{\gamma}^{a}$ through vierbein as

$$
\gamma^{\mu}=h_{a}^{\mu} \tilde{\gamma}^{a}
$$

So, Dirac equation in (1.1) is written as

$$
i\left[\tilde{\gamma}^{0}\left(\partial_{o}+\frac{9}{4} \frac{\dot{a}}{a}\right)+a^{-1}\left(\bar{\gamma}^{1} \partial_{1}+\bar{\gamma}^{2} \partial_{2}+\bar{\gamma}^{3} \partial_{3}\right)+a \bar{\gamma}^{5} \partial_{5}\right] \Psi_{5}-m_{5} \Psi_{5}=0,\left(\dot{a}=\partial_{0} a\right)
$$


The internal space is compactified, so

$$
\Psi_{5}(x, y)=\sum_{n=-\infty}^{\infty} \phi_{n}(y) \Psi_{4}^{(n)}(x)
$$

where $\Psi_{4}^{(n)}(x)$ are 4-dim. spinor fields in nth mode and $\phi_{n}(y)$ are harmonic functions which obey the equation[4]

$$
i \tilde{\gamma}^{5} \partial_{5} \phi_{n}(y) \psi_{4}^{(n)}=\frac{n}{R} \phi_{n}(y) \psi_{4}^{(n)}
$$

$\mathrm{R}$ is the compactification scale. $\phi_{n}$ is normalised as

$$
\phi_{n} \phi_{m}=\delta_{n m}
$$

Connecting (2.8) and (2.10)

$$
\left[i\left\{\tilde{\gamma}^{0}\left(\partial_{0}+\frac{9 \dot{a}}{4 a}\right)+a^{-1}\left(\tilde{\gamma}^{1} \partial_{1}+\tilde{\gamma}^{2} \partial_{2}+\bar{\gamma}^{3} \partial_{3}\right)\right\}-\left(m_{5}-\frac{a n}{R}\right)\right] \Psi_{4}^{n}=0
$$

(2.11) shows that effective mass for $\Psi_{4}^{n}$ is

$$
M=m_{5}-\frac{a n}{R}=m_{5}-a n M_{c}
$$

where $R^{-1}=M_{c}$ is the compactification mass.

The Extra dimension $y$ which is assumed to be a circle of radius $\mathrm{R}$, has the range

$$
0 \leq y \leq 2 \pi R
$$

The distance around the extra dimension is given by [13]

$$
\delta_{5}=\int_{o}^{2 \pi R} d y \sqrt{-g_{55}}=\frac{2 \pi R}{a(t)}
$$

which is time-dependent and decreases as $a(t)$ increases.

At $t=t_{c}$ (compactification time), it is assumed that

$$
\frac{2 \pi R}{a\left(t_{c}\right)} \sim L_{P} \quad(\text { PlankLength })
$$

For realistic fermions, $M \simeq 0$. So, as a particular time $t=t_{P}$

$$
m_{5} \simeq \frac{n a\left(t_{p}\right)}{R}
$$


Connecting (2.15) and (2.16)

$$
m_{5} \simeq \frac{2 \pi n a\left(t_{p}\right) M_{P}}{a\left(t_{c}\right)}
$$

where $M_{P}$ is the Planck mass and is equal to $\left(L_{P}\right)^{-1}$

Hence from (2.17)

$$
n \simeq \frac{m_{5} a\left(t_{c}\right)}{2 \pi a\left(t_{p}\right) M_{P}} \leq \frac{m_{5}}{2 \pi M_{P}}
$$

as $a\left(t_{p}\right) \gtrsim a\left(t_{c}\right)$ in the expanding 4-dim. universe. So (2.18) puts a constraint on modes n. Also (2.18) states that if one gets realistic 4-dim. fermion from 5-dim fermion as a result of compactification of the extra dimension, number of physically allowed modes depends on $m_{5}$. For example, $n=0,1$ if $m_{5}=2 \pi M_{p} ; n=0,1,2$ if $m_{5}=4 \pi M_{p}$; $n=0,1,2, \ldots . .(r-1), r$ if $m_{5}=2 \pi r M_{p}$ where $r$ is positive integer. From $(2.16)$

$$
a\left(t_{p}\right) \simeq \frac{m_{5} R}{n}
$$

So, one gets

$$
a\left(t_{p}\right) \simeq \frac{2 \pi r M_{p} R}{n}
$$

where $n=0,1,2, \ldots(r-1), r$. Now it is interesting to see that if $a\left(t_{p 1}\right)=a_{1}$ when $n=r$ and $a\left(t_{p 2}\right)=a_{2}$ when $n=r-1, a_{2}>a_{1}$. It means that realistic fermions may be obtained in higher modes earlier than those in lower modes during the course of expansion of the obsevable 4-dim. universe, as $t_{p 1}<t_{p 2}$.

\section{Solution of Dirac Equation}

Substituting $\Psi$ defined as

$$
\psi_{4}^{(n)}=a^{-\frac{9}{4}} \Psi
$$

(2.11) is re-written as

$$
\left[\left(\tilde{\gamma}^{0} \partial_{\tau}+\tilde{\gamma}^{1} \partial_{1}+\tilde{\gamma}^{2} \partial_{2}+\tilde{\gamma}^{3} \partial_{3}\right)+i a\left(m_{5}-a n M_{c}\right)\right] \Psi=0
$$

with

$$
\tau=\int^{t} \frac{d t^{\prime}}{a\left(t^{\prime}\right)}
$$

Writing

$$
\Psi=\sum_{k} \Psi_{k}=(2 \pi)^{-\frac{3}{2}} \exp (i k x)\left[\begin{array}{c}
f_{I}(\vec{k}, \tau) \\
f_{I I}(\vec{k}, \tau)
\end{array}\right]
$$


One gets two coupled equations

$$
\begin{aligned}
& {\left[\partial_{\tau}+i a\left(m_{5}-a n M_{c}\right)\right] f_{I}+i(k . \sigma) f_{I I}=0} \\
& {\left[\partial_{\tau}-i a\left(m_{5}-a n M_{c}\right)\right] f_{I I}+i(k . \sigma) f_{I}=0}
\end{aligned}
$$

where $\sigma_{1}, \sigma_{2}, \sigma_{3}$ are pauli matrices.

From (3.5a)

$$
f_{I I}=i k^{-2}(k . \sigma)\left[\partial_{\tau}+i a\left(m_{5}-a n M_{c}\right)\right] f_{I}
$$

Connecting (3.5b)and (3.6)

$$
f_{I}^{\prime \prime}+\left[k^{2}+a^{2}\left(m_{5}-a n M_{c}\right)^{2}+i \partial_{\tau}\left\{a\left(m_{5}-a n M_{c}\right)\right\}\right] f_{I}=0
$$

where prime $(/)$ denotes differentiation with respect to $\tau$.

The norm for $\psi_{5}$ is defined at $\tau=$ constant hypersurface as

$$
\left(\psi_{5 k s}, \psi_{5 k^{\prime} s^{\prime}}\right)=\int_{\tau} \sqrt{\left|g_{5}\right|} d^{3} x d y \bar{\psi}_{5 k s} \gamma^{0} \psi_{5 k^{\prime} s^{\prime}}
$$

where $g_{5}$ is the determinant of 5 -dim. metric tensor.

Connecting (2.9) and (3.8)

$$
\left(\psi_{5 k s}, \psi_{5 k^{\prime} s^{\prime}}\right)=\int_{\tau} \sqrt{\left|g_{5}\right|} d^{3} x d y \sum_{n} \bar{\psi}_{4 k s}^{n}(x) \bar{\phi}_{n}(y) \gamma^{0} \sum_{n^{\prime}} \phi_{n^{\prime}}(y) \psi_{4 k^{\prime} s^{\prime}}^{n^{\prime}}(x)
$$

which yields, on integration over extra dimension $y$ having the range $0 \leq y \leq 2 \pi R$

$$
\left(\psi_{5 k s}, \psi_{5 k^{\prime} s^{\prime}}\right)=2 \pi R \int_{\tau} \sqrt{\left|g_{5}\right|} d^{3} x \sum_{n} \bar{\psi}_{4 k s}^{n} \gamma^{0} \psi_{4 k^{\prime} s^{\prime}}^{n}
$$

The normalization constants are determined using the prescription that in the flat space limit

$$
\left(\psi_{5 k s}, \psi_{5 k^{\prime} s^{\prime}}\right) \longrightarrow \delta_{s s^{\prime}} \delta^{3}\left(k-k^{\prime}\right)
$$

or,

$$
2 \pi R \int_{\tau} \sqrt{\left|g_{5}\right|} d^{3} x \sum_{n} \bar{\psi}_{4 k s}^{n} \gamma^{0} \psi_{4 k^{\prime} s^{\prime}}^{n} \longrightarrow \delta_{s s^{\prime}} \delta^{3}\left(k-k^{\prime}\right)
$$

Now, some explicit examples of solutions of Dirac equation for $\psi_{4}^{n}$ utilising the above mentioned procedure of normalization are given as under for different $a(t)$.

$$
\underline{3(A) \quad a(t) \simeq t^{\frac{1}{2}}}
$$


From (3.3)

$$
\tau=2 t^{\frac{1}{2}} \text { and } a(t)=\frac{1}{2} \tau
$$

so (3.7) is written as

$$
f_{I}^{\prime \prime}+\left[k^{2}+\frac{\tau^{2}}{4}\left(m_{5}-\frac{n M_{c} \tau}{2}\right)^{2}+\frac{i}{2} \partial_{\tau}\left\{\tau\left(m_{5}-\frac{n M_{c} \tau}{2}\right)\right\}\right] f_{I}=0
$$

(3.14) can be approximated in two different ways:

Case I: When $m_{5}>>\frac{1}{2} n M_{c} \tau$ (3.14) is approximated as

$$
f_{I}^{\prime \prime}+\left[k^{2}+\frac{i m_{5}}{2}-\frac{i n M_{c} \tau}{4}+\frac{m_{5}^{2} \tau^{2}}{4}\right] f_{I}=0
$$

which has exact solution

$$
\begin{aligned}
f_{I}= & \exp \left[ \pm \frac{n M_{c}}{4 m_{5}} \tau \mp \frac{i m_{5}}{4} \tau^{2}\right]\left[N_{1}{ }_{1} F_{1}\left(a, \frac{1}{2}, X\right)+\right. \\
& \left.N_{2} \frac{e^{i(6 r+1) \frac{\pi}{4}}}{\sqrt{2 m_{5}}}\left(\frac{n M_{c}}{2 m_{5}}-i m_{5} \tau\right){ }_{1} F_{1}\left(\frac{1}{2}+a, \frac{3}{2}, X\right)\right]
\end{aligned}
$$

where

$$
\begin{aligned}
a & = \pm \frac{i}{m_{5}}\left(\frac{n^{2} M_{c}^{2}}{32 m_{5}^{4}}+\frac{k^{2}}{2 m_{5}^{2}}\right), \\
X & = \pm \frac{i}{2 m_{5}}\left(\frac{n M_{c}}{2 m_{5}}-i m_{5} \tau\right)^{2},
\end{aligned}
$$

${ }_{1} F_{1}(a, c, X)$ is confluent hypergeometric function and $r=0,1,2, \ldots$

Connecting (3.6)and(3.16)

$$
\begin{aligned}
& f_{I I}=\frac{i(k . \sigma)}{k^{2}} \exp \left[ \pm \frac{n M_{c}}{4 m_{5}} \tau \mp \frac{i m_{5}}{4} \tau^{2}\right] \times \\
& {\left[N_{3}\left\{\left( \pm \frac{n M_{c}}{4 m_{5}} \mp \frac{i m_{5}}{2} \tau+\frac{i m_{5}}{2} \tau-\frac{i n \tau^{2} M_{c}}{4}\right){ }_{1} F_{1}\left(a, \frac{1}{2}, X\right)+2 a X^{\prime}{ }_{1} F_{1}\left(1+a, \frac{3}{2}, X\right)\right\}+\right.} \\
& N_{4} e^{i(6 r+1) \frac{\pi}{4}}\left\{\left(\frac{n M_{c}}{2 m_{5}}-i m_{5} \tau\right)\left( \pm \frac{n M_{c}}{4 m_{5}} \mp \frac{i m_{5}}{2} \tau+\frac{i m_{5}}{2} \tau-\frac{i n \tau^{2} M_{c}}{4}\right){ }_{1} F_{1}\left(\frac{1}{2}+a, \frac{3}{2}, X\right)-\right. \\
& \left.\left.i \sqrt{\frac{m_{5}}{2}}{ }_{1} F_{1}\left(\frac{1}{2}+a, \frac{3}{2}, X\right)+\frac{(1+2 a)}{3} X^{\prime} \frac{\left(\frac{n M_{c}}{2 m_{5}}-i m_{5} \tau\right)}{\sqrt{2 m_{5}}}{ }_{1} F_{1}\left(\frac{3}{2}+a, \frac{5}{2}, X\right)\right\}\right]
\end{aligned}
$$

Corresponding to $f_{I}$ and $f_{I I}, \psi_{k 4 I s}^{n}$ and $\psi_{k 4 I I s}^{n}$ are written as

$$
\begin{gathered}
\psi_{k 4 I s}^{n}=(2 \pi)^{-\frac{3}{2}}\left(\frac{\tau}{2}\right)^{-\frac{9}{4}} \exp \left[i k x \pm \frac{n M_{c}}{4 m_{5}} \tau \mp \frac{i m_{5} \tau^{2}}{4}\right] \times\left[N_{1} u_{s i} F_{1}\left(a, \frac{1}{2}, X\right)+\right. \\
\left.N_{2} \hat{u}_{s} e^{i(6 r+1) \pi / 4} \frac{\left(\frac{n M_{c}}{2 m_{5}}-i m_{5} \tau\right)}{\sqrt{2 m_{5}}}{ }_{1} F_{1}\left(\frac{1}{2}+a, \frac{3}{2}, X\right)\right]
\end{gathered}
$$




$$
\begin{aligned}
& \psi_{k 4 I I s}^{n}=(2 \pi)^{-\frac{3}{2}}\left(\frac{\tau}{2}\right)^{-\frac{9}{4}} \frac{i(k \cdot \sigma)}{k^{2}} \exp \left[i k . x \pm \frac{n M_{c}}{4 m_{5}} \tau \mp \frac{i m_{5} \tau^{2}}{4}\right] \times \\
& {\left[N_{3} u_{s}\left\{\left( \pm \frac{n M_{c}}{4 m_{5}} \mp \frac{i m_{5}}{2} \tau+\frac{i m_{5}}{2} \tau-\frac{i n \tau^{2} M_{c}}{4}\right){ }_{1} F_{1}\left(a, \frac{1}{2}, X\right)+2 a X^{\prime}{ }_{1} F_{1}\left(1+a, \frac{3}{2}, X\right)\right\}+\right.} \\
& N_{4} \hat{u}_{s} e^{i(6 r+1) \pi / 4}\left\{\frac{\left(\frac{n M_{c}}{2 m_{5}}-i m_{5} \tau\right)}{\sqrt{2 m_{5}}}\left( \pm \frac{n M_{c}}{4 m_{5}} \mp \frac{i m_{5}}{2} \tau+\frac{i m_{5}}{2} \tau-\frac{i n \tau^{2} M_{c}}{4}\right)_{1} F_{1}\left(\frac{1}{2}+a, \frac{3}{2}, X\right)-\right. \\
& \left.\left.i \sqrt{\frac{m_{5}}{2}}{ }_{1} F_{1}\left(\frac{1}{2}+a, \frac{3}{2}, X\right)+\frac{(1+2 a)}{3} X^{\prime} \frac{\left(\frac{n M_{c}}{2 m_{5}}-i m_{5} \tau\right)}{\sqrt{2 m_{5}}}{ }_{1} F_{1}\left(\frac{3}{2}+a, \frac{5}{2}, X\right)\right\}\right]
\end{aligned}
$$

where $u_{s}$ and $\hat{u}_{s}$ are column matrices (with spin quantum number $\mathrm{s}= \pm 1$ ) given as

$$
\begin{array}{rlr}
\hat{u}_{1}=\left(\begin{array}{l}
1 \\
0 \\
0 \\
0
\end{array}\right), & \hat{u}_{-1}=\left(\begin{array}{l}
0 \\
1 \\
0 \\
0
\end{array}\right) \\
u_{1}=\left(\begin{array}{c}
0 \\
0 \\
-k_{3} \\
-k_{1}-i k_{2}
\end{array}\right) \text { and } & u_{-1}=\left(\begin{array}{c}
0 \\
0 \\
-k_{1}+i k_{2} \\
k_{3}
\end{array}\right)
\end{array}
$$

Normalization constants $N_{1}, N_{2}, N_{3}$ and $N_{4}$ are determined through (3.12) as

$$
\begin{aligned}
& N_{1}=\frac{\sqrt{M_{c}} \exp \left(\mp n M_{c} / 2 m_{5}\right)}{2 k \sqrt{\pi}\left|{ }_{1} F_{1}\left(a, \frac{1}{2}, \bar{X}\right)\right|} \\
& N_{2}=\frac{\sqrt{M_{c}} \exp \left(\mp n M_{c} / 2 m_{5}\right)}{2 \sqrt{\pi} \sqrt{\frac{n^{2} M_{c}^{2}+16 m_{5}^{2}}{8 m_{5}^{3}}}\left|{ }_{1} F_{1}\left(a, \frac{1}{2}+a, \frac{3}{2}, \bar{X}\right)\right|} \\
& N_{3}=\frac{\sqrt{M_{c}} \exp \left(\mp n M_{c} / 2 m_{5}\right)}{2 \sqrt{\pi} s_{1}} \\
& N_{4}=\frac{\sqrt{M_{c}} \exp \left(\mp n M_{c} / 2 m_{5}\right)}{2 k \sqrt{\pi} s_{2}}
\end{aligned}
$$

where

$$
\begin{gathered}
\bar{X}= \pm\left(\frac{n M_{c}}{2 m_{5}}-2 i m_{5}\right) \\
S_{1}=\left|\left( \pm \frac{n M_{c}}{4 m_{5}} \mp i m_{5}+i m_{5}-i n M_{c}\right)_{1} F_{1}\left(a, \frac{1}{2}, \bar{X}\right) \pm 2 a\left(\frac{n M_{c}}{2 m_{5}}-i m_{5}\right)_{1} F_{1}\left(1+a, \frac{3}{2}, \bar{X}\right)\right|
\end{gathered}
$$


and

$$
\begin{aligned}
S_{2}= & \mid \frac{\left(\frac{n M_{c}}{2 m_{5}}-2 i m_{5}\right)}{\sqrt{2 m_{5}}}\left( \pm \frac{n M_{c}}{4 m_{5}} \mp i m_{5}+i m_{5}-i n M_{c}\right)_{1} F_{1}\left(\frac{1}{2}+a, \frac{3}{2}, \bar{X}\right)- \\
& i \sqrt{\frac{m_{5}}{2}}{ }_{1} F_{1}\left(\frac{1}{2}+a, \frac{3}{2}, \bar{X}\right)+\frac{(1+2 a)}{3} \frac{\left(\frac{n M_{c}}{2 m_{5}}-2 i m_{5}\right)}{\sqrt{2 m_{5}}}{ }_{1} F_{1}\left(\frac{3}{2}+a, \frac{5}{2}, \bar{X}\right) \mid
\end{aligned}
$$

Case II When $m_{5} \gtrsim \frac{n \tau}{2} M_{c}$, a new variable $\tau^{\prime}$ is defined as $\tau^{\prime}=m_{5}-\frac{n M_{c}}{2} \tau$. Now (3.14) is written as

$$
\frac{d^{2} f_{I}}{d \tau^{\prime 2}}+\frac{4}{n^{2} M_{c}^{2}}\left[k^{2}+\frac{1}{n^{2} M_{c}^{2}}\left(m_{5}-\tau^{\prime}\right)^{2} \tau^{\prime 2}-\frac{i}{2} \frac{d}{d \tau^{\prime}}\left\{\tau^{\prime}\left(m_{5}-\tau^{\prime}\right)\right\}\right] f_{I}=0
$$

Since $m_{5}>>\tau^{\prime}$, so one gets

$$
\frac{d^{2} f_{I}}{d \tau^{\prime 2}}+\left[\frac{4}{n^{2} M_{c}^{2}}\left(k^{2}-\frac{i m_{5}}{2}\right)+\frac{4 m_{5}^{2}}{n^{4} M_{c}^{4}} \tau^{\prime 2}\right] f_{I}=0
$$

having the exact solution

$$
f_{I}=\exp \left(-\frac{z}{2}\right)\left[\tilde{N}_{1}{ }_{1} F_{1}\left(k, \frac{1}{2}, z\right)+\tilde{N}_{2} z^{1 / 2}{ }_{1} F_{1}\left(k+\frac{1}{2}, \frac{3}{2}, z\right)\right]
$$

where

$$
z=\frac{i m_{5}}{2}\left(\tau-\frac{2 m_{5}}{n M_{c}}\right)^{2}
$$

and

$$
4 k=1+\frac{2 i}{m_{5}}\left(k^{2}-\frac{i m_{5}}{2}\right)
$$

Connecting (3.6) and (3.22)

$$
\begin{aligned}
& f_{I I}=\frac{i(k \cdot \sigma)}{k^{2}} \exp \left(-\frac{z}{2}\right)\left[\tilde { N } _ { 3 } \left\{\left(\frac{1}{2} z^{\prime}-\frac{i n M_{c} \tau^{2}}{4}+\frac{i m_{5} \tau}{2}\right){ }_{1} F_{1}\left(k, \frac{1}{2}, z\right)+\right.\right. \\
& \left.2 k z^{\prime}{ }_{1} F_{1}\left(k+1, \frac{3}{2}, z\right)\right\}+\tilde{N}_{4}\left\{z^{1 / 2}\left(\frac{1}{2} z^{\prime}-\frac{i n M_{c}}{4} \tau^{2}+\frac{i m_{5} \tau}{2}+\frac{z^{\prime}}{2 z}\right){ }_{1} F_{1}\left(k+\frac{1}{2}, \frac{3}{2}, z\right)\right. \\
& \left.\left.+\frac{(2 k+1)}{3} z^{\prime} z^{1 / 2}{ }_{1} F_{1}\left(k+\frac{3}{2}, \frac{5}{2}, z\right)\right\}\right]
\end{aligned}
$$

Corresponding to $f_{I}$ and $f_{I I}, \psi_{k 4 I s}^{n}$ and $\psi_{k 4 I I s}^{n}$ as are written as

$$
\begin{aligned}
& \psi_{k 4 I s}^{n}=(2 \pi)^{-3 / 2} \exp [i k \cdot x]\left(\frac{\tau}{2}\right)^{-9 / 4}\left[\tilde{N}_{1} u_{s} F_{1}\left(k, \frac{1}{2}, z\right)+\tilde{N}_{2} \hat{u}_{s} z^{1 / 2}{ }_{1} F_{1}\left(k+\frac{1}{2}, \frac{3}{2}, z\right)\right] \\
& \psi_{k 4 I I s}^{n}=(2 \pi)^{-3 / 2} \exp [i k \cdot x]\left(\frac{\tau}{2}\right)^{-9 / 4} e^{-z / 2} \frac{i(k \cdot \sigma)}{k^{2}} \times \\
& {\left[\tilde{N}_{3} u_{s}\left\{\left(\frac{1}{2} z^{\prime}-\frac{i n M_{c} \tau^{2}}{4}+\frac{i n M_{5} \tau}{2}\right){ }_{1} F_{1}\left(k, \frac{1}{2}, z\right)+2 k z^{\prime}{ }_{1} F_{1}\left(k+1, \frac{3}{2}, z\right)\right\}\right.} \\
& +\tilde{N}_{4} \hat{u}_{s}\left\{z^{1 / 2}\left(\frac{1}{2} z^{\prime}-\frac{i n M_{c} \tau^{2}}{4}+\frac{i n M_{5} \tau}{2}+\frac{z^{\prime} z^{-1}}{2}\right){ }_{1} F_{1}\left(k+\frac{1}{2}, \frac{3}{2}, z\right)+\right. \\
& \left.\left.\quad \frac{(2 k+1)}{3} z^{\prime} z^{\frac{1}{2}}{ }_{1} F_{1}\left(k+\frac{3}{2}, \frac{5}{2}, z\right)\right\}\right]
\end{aligned}
$$


On normalizing these solutions

$$
\begin{aligned}
& \tilde{N}_{1}=\sqrt{M_{c}}\left[2 k \sqrt{\pi}\left|{ }_{1} F_{1}\left(k, \frac{1}{2}, \frac{2 i m_{5}}{n^{2} M_{c}^{2}}\left(n M_{c}-m_{5}\right)^{2}\right)\right|\right]^{-1} \\
& \tilde{N}_{2}=n \sqrt{M_{c}}\left[2 \sqrt{\pi}\left(n M_{c}-m_{5}\right) \sqrt{2 m_{5}}\left|{ }_{1} F_{1}\left(k+\frac{1}{2}, \frac{3}{2}, \frac{2 i m_{5}}{n^{2} M_{c}^{2}}\left(n M_{c}-m_{5}\right)^{2}\right)\right|\right]^{-1} \\
& \tilde{N}_{3}=\frac{n \sqrt{M_{c}}}{2 \sqrt{\pi}}\left(\tilde{s_{1}}\right)^{-1} \\
& \tilde{N}_{4}=\frac{k n \sqrt{M_{c}}}{2 \sqrt{n}}\left(\tilde{s_{2}}\right)^{-1}
\end{aligned}
$$

where

$$
\begin{aligned}
\tilde{S}_{1}= & \mid\left\{i m_{5}\left(n M_{c}-m_{5}\right)-i n^{2} M_{c}^{2}+i n m_{5} M_{c}\right\}_{1} F_{1}\left(k, \frac{1}{2}, \frac{2 i m_{5}}{n^{2} M_{c}^{2}}\left(n M_{c}-m_{5}\right)^{2}\right. \\
& +4 i k m_{5}\left(n M_{c}-m_{5}\right)_{1} F_{1}\left(k+1, \frac{3}{2}, \frac{2 i m_{5}}{n^{2} M_{c}^{2}}\left(n M_{c}-m_{5}\right)^{2}\right) \mid
\end{aligned}
$$

and

$$
\begin{gathered}
\left(\tilde{S}_{2}\right)^{2}=m_{5}\left(1-\frac{m_{5}}{n M_{c}}\right) \mid\left\{\frac{i m_{5}\left(n M_{c}-m_{5}\right)}{n M_{c}}-i n M_{c}+i m_{5}+\frac{n M_{c}}{2\left(n M_{c}-m_{5}\right.}\right\} \times \\
{ }_{1} F_{1}\left(k+\frac{1}{2}, \frac{3}{2}, \frac{2 i m_{5}}{n^{2} M_{c}^{2}}\left(n M_{c}-m_{5}\right)^{2}\right)+ \\
\frac{(2 k+1)}{3} \frac{2 i m_{5}\left(n M_{c}-m_{5}\right)}{n M_{c}}{ }_{1} F_{1}\left(k+\frac{3}{2}, \frac{5}{2}, \frac{2 i m_{5}}{n^{2} M_{c}^{2}}\left(n M_{c}-m_{5}\right)^{2}\right) \mid \\
\underline{3 B . \quad a(t) \simeq t}
\end{gathered}
$$

From (3.3), in this case

$$
\tau=\ln t, \quad a(t)=\exp (\tau)
$$

Connecting (3.7) and (3.26)

$$
f_{I}^{\prime \prime}+\left[k^{2}+e^{2 \tau}\left(m_{5}-n M_{c} e^{\tau}\right)^{2}+i e^{\tau}\left(m_{5}-n M_{c} e^{\tau}\right)-i n M_{c} e^{2 \tau}\right] f_{I}=0
$$

Case 1 When $m_{5}>>n M_{c} e^{\tau},(3.27)$ is approximated as

$$
f_{I}^{\prime \prime}+\left[k^{2}+i m_{5} e^{\tau}+\left(m_{5}^{2}-i n M_{c}\right) e^{2 \tau}\right] f_{I}=0
$$

which yields the exact solutions

$$
\begin{aligned}
& f_{I}(\tau)=\exp \left[\mp i k \tau+\left(i n M_{c}-m_{5}^{2}\right) e^{\tau}\right] \times\left[c_{1}{ }_{1} F_{1}\left(\frac{2 l l^{\prime}+i m_{5}}{2 l^{\prime}}, 2 l,-\frac{e^{\tau}}{2 l^{\prime}}\right)+\right. \\
& \left.c_{2}\left(-\frac{e^{\tau}}{2 l^{\prime}}\right)^{1-2 l}{ }_{1} F_{1}\left(\frac{2 l^{\prime}-2 l l^{\prime}+i m_{5}}{2 l^{\prime}}, 2-2 l,-\frac{e^{\tau}}{2 l^{\prime}}\right)\right]
\end{aligned}
$$


where

$$
l=\frac{1}{2}[1 \pm i 2 k], \quad l^{\prime}=i n M_{c}-m_{5}^{2}
$$

Connecting (3.6) and (3.29)

$$
f_{I I}(\tau)=\frac{i(k . \sigma)}{k^{2}} \exp \left[\mp i k \tau+\left(i n M_{c}-m_{5}^{2}\right) e^{\tau}\right]\left[c_{3} X(\tau)+c_{4} Y(\tau)\right]
$$

where

$$
\begin{aligned}
X(\tau) & =\left\{\mp i k+i n M_{c}-m_{5}^{2}-i e^{\tau}\left(n M_{c} e^{\tau}-m_{5}\right)\right\}_{1} F_{1}\left(\frac{2 l l^{\prime}+i m_{5}}{2 l^{\prime}}, 2 l,-\frac{e^{\tau}}{2 l^{\prime}}\right) \\
& -\frac{\left(2 l l^{\prime}+i m_{5}\right)}{8 l l^{\prime 2}}{ }_{1} F_{1}\left(\frac{2 l l^{\prime}+i m_{5}+2 l^{\prime}}{2 l^{\prime}}, 1+2 l,-\frac{e^{\tau}}{2 l^{\prime}}\right)
\end{aligned}
$$

and

$$
\begin{aligned}
Y(\tau) & =\left(-\frac{e^{\tau}}{2 l^{\prime}}\right)^{1-2 l}\left[\left\{1-2 l \mp i k+i n M_{c}-m_{5}^{2}-i e^{\tau}\left(n M_{c} e^{\tau}-m_{5}\right)\right\} \times\right. \\
{ }_{1} & F_{1}\left(\frac{2 l^{\prime}-2 l l^{\prime}+i m_{5}}{2 l^{\prime}}, 2-2 l,-\frac{e^{\tau}}{2 l^{\prime}}\right) \\
& \left.-\frac{\left(2 l^{\prime}-2 l l^{\prime}+i m_{5}\right)}{8 l^{\prime 2}(1-l)}{ }_{1} F_{1}\left(\frac{4 l^{\prime}-2 l l^{\prime}+i m_{5}}{2 l^{\prime}}, 3-2 l,-\frac{e^{\tau}}{2 l^{\prime}}\right)\right]
\end{aligned}
$$

So,

$$
\begin{aligned}
\psi_{k 4 I s}^{n} & =(2 \pi)^{-3 / 2} \exp \left[i k . x-\frac{9 \tau}{4} \mp i k \tau+\left(i n M_{c}-m_{5}^{2}\right) e^{\tau}\right] \times \\
& {\left[c_{1} u_{s}{ }_{1} F_{1}\left(\frac{2 l l^{\prime}+i m_{5}}{2 l^{\prime}}, 2 l,-\frac{e^{\tau}}{2 l^{\prime}}\right)\right.} \\
& \left.+c_{2} \hat{u}_{s}\left(-\frac{e^{\tau}}{2 l^{\prime}}\right)^{1-2 l}{ }_{1} F_{1}\left(\frac{2 l^{\prime}-2 l l^{\prime}+i m_{5}}{2 l^{\prime}}, 2-2 l,-\frac{e^{\tau}}{2 l^{\prime}}\right)\right]
\end{aligned}
$$

and

$$
\begin{gathered}
\psi_{k 4 I I s}^{n}=\frac{i(k . \sigma)}{k^{2}}(2 \pi)^{-3 / 2} \exp \left[i k . x-\frac{9 \tau}{4} \mp i k \tau+\left(i n M_{c}-m_{5}^{2}\right) e^{\tau}\right] \times \\
{\left[c_{3} u_{s} X(\tau)+c_{4} \hat{u}_{s} Y(\tau)\right]}
\end{gathered}
$$

On normalising these solutions

$$
\begin{aligned}
c_{1} & =\frac{\sqrt{M_{c}} \exp \left(m_{5}^{2}\right)}{2 \sqrt{\pi} k\left|{ }_{1} F_{1}\left(\frac{2 l l^{\prime}+i m_{5}}{2 l^{\prime}}, 2 l,-\frac{1}{2 l^{\prime}}\right)\right|} \\
c_{2} & =\frac{\sqrt{M_{c}}\left(2 l^{\prime}\right)^{1-2 l} \exp \left(m_{5}^{2}\right)}{2 \sqrt{\pi}\left|{ }_{1} F_{1}\left(\frac{2 l^{\prime}-2 l l^{\prime}+i m_{5}}{2 l^{\prime}}, 2-2 l,-\frac{1}{2 l^{\prime}}\right)\right|} \\
c_{3} & =\frac{\sqrt{M_{c}} \exp \left(m_{5}^{2}\right)}{2 \sqrt{\pi}|X(0)|}
\end{aligned}
$$




$$
c_{4}=\frac{\sqrt{M_{c}} \exp \left(m_{5}^{2}\right)}{2 \sqrt{\pi}|Y(0)|}
$$

where $X(\tau)$ and $Y(\tau)$ are defined as in (3.30)

Case II. When $m_{5} \gtrsim n M_{c} e^{\tau},(3.27)$ is approximated a

$$
f_{I}^{\prime \prime}+\left[k^{2}-i n M_{c} e^{2 \tau}\right] f_{I}=0
$$

which integrates to

$$
\begin{aligned}
f_{I}= & \exp \left( \pm i k \tau+l^{\prime} e^{\tau}\right)\left[\tilde{c}_{1}{ }_{1} F_{1}\left(\frac{2 l l^{\prime}-i n M_{c}}{2 l^{\prime}}, 2 l,-2 l^{\prime} e^{\tau}\right)+\right. \\
& \left.\tilde{c}_{2}\left(-2 l^{\prime}\right)^{1-2 l} e^{\tau(1-2 l)}{ }_{1} F_{1}\left(\frac{2 l^{\prime}-2 l l^{\prime}-i n M_{c}}{2 l^{\prime}}, 2-2 l,-2 l^{\prime} e^{\tau}\right)\right]
\end{aligned}
$$

where $L=\frac{1}{2}[1 \pm 2 i k]$ and $L^{\prime}=\sqrt{n M_{c}} \times \exp \left[(2 r+1) \frac{\pi}{4}\right]$ with $r=0,1,2, \ldots$ (3.6) and (3.34) yield

$$
f_{I I}=\frac{i(k . \sigma)}{k^{2}} \exp \left( \pm i k \tau+L^{\prime} e^{\tau}\right)\left[\tilde{c}_{3} \tilde{X}(\tau) \quad \tilde{c}_{4} \tilde{Y}(\tau)\right]
$$

where

$$
\begin{aligned}
\tilde{X}(\tau) & =\left\{ \pm i k \tau+L^{\prime} e^{\tau}+i e^{\tau}\left(m_{5}-n M_{c} e^{\tau}\right)\right\}_{1} F_{1}\left(\frac{2 L L^{\prime}-i n M_{c}}{2 L^{\prime}}, 2 L,-2 L^{\prime} e^{\tau}\right) \\
& -\frac{\left(2 L L^{\prime}-i n M_{c}\right)}{2 L} e^{\tau}{ }_{1} F_{1}\left(\frac{2 L^{\prime}+2 L L^{\prime}-i n M_{c}}{2 L^{\prime}}, 1+2 L,-2 L^{\prime} e^{\tau}\right)
\end{aligned}
$$

and

$$
\begin{aligned}
\tilde{Y}(\tau) & =\left\{ \pm i k \tau+L^{\prime} e^{\tau}+\left(-2 L^{\prime}\right)^{1-2 L}(2-2 L) e^{\tau(1-2 l)}+i e^{\tau}\left(m_{5}-i n M_{c} e^{\tau}\right)\right\} \times \\
& { }_{1} F_{1}\left(\frac{2 L^{\prime}-2 L L^{\prime}-i n M_{c}}{2 L^{\prime}}, 2-2 L,-2 L^{\prime} e^{\tau}\right)+ \\
& \left(-2 L^{\prime}\right)^{1-2 L} \frac{\left(2 L^{\prime}-2 L L^{\prime}-i n M_{c}\right)}{2(L-1)} e^{2(1-L) \tau} \times \\
& { }_{1} F_{1}\left(\frac{4 L^{\prime}-2 L L^{\prime}-i n M_{c}}{2 L^{\prime}}, 3-2 L,-2 L^{\prime} e^{\tau}\right)
\end{aligned}
$$

So,

$$
\begin{aligned}
\psi_{k 4 I s}^{n} & =(2 \pi)^{-3 / 2} \exp \left[i k x-\frac{9 \tau}{4} \pm i k \tau+L^{\prime} e^{\tau}\right] \times \\
& {\left[\tilde{c}_{1} u_{s}{ }_{1} F_{1}\left(\frac{2 L L^{\prime}-i n M_{5}}{2 L^{\prime}}, 2 L,-2 L^{\prime} e^{\tau}\right)+\right.} \\
& \left.\tilde{c}_{2} \hat{u}_{s}\left(-2 L^{\prime}\right)^{1-2 L} e^{\tau(1-2 L)}{ }_{1} F_{1}\left(\frac{2 L^{\prime}-2 L L^{\prime}-i n M_{c}}{2 L^{\prime}}, 2-2 L,-2 L^{\prime} e^{\tau}\right)\right]
\end{aligned}
$$


and

$$
\begin{aligned}
\psi_{k 4 I I s}^{n}= & \frac{i(k . \sigma)}{k^{2}}(2 \pi)^{-3 / 2} \exp \left[i k \cdot x-\frac{9 \tau}{4} \pm i k \tau+L^{\prime} e^{\tau}\right] \times \\
& {\left[\tilde{c}_{3} u_{s} \tilde{X}(\tau)+\tilde{c}_{4} \hat{u}_{s} \tilde{Y}(\tau)\right] }
\end{aligned}
$$

where $\tilde{X}(\tau)$ and $\tilde{Y}(\tau)$ are defined in (3.35)

On normalizing the above solutions

$$
\begin{gathered}
\tilde{c}_{1}=\frac{e^{-L^{\prime}} \sqrt{M_{c}}}{k \sqrt{2 \pi}\left|{ }_{1} F_{1}\left(\frac{2 L L^{\prime}-i n M_{c}}{2 L^{\prime}}, 2 L,-2 L^{\prime}\right)\right|} \\
\tilde{c}_{2}=\frac{e^{-L^{\prime}} \sqrt{M_{c}}\left(-2 L^{\prime}\right)^{(L-1)}}{\sqrt{2 \pi}\left|{ }_{1} F_{1}\left(\frac{2 L^{\prime}-2 L L^{\prime}-i n M_{c}}{2 L^{\prime}}, 2-2 L,-2 L^{\prime}\right)\right|} \\
\tilde{c}_{3}=\frac{e^{-L^{\prime}} \sqrt{M_{c}}}{\sqrt{2 \pi}|\tilde{X}(0)|} \\
\tilde{c}_{4}=\frac{k e^{-L^{\prime}} \sqrt{M_{c}}}{\sqrt{2 \pi}|\tilde{Y}(0)|} \\
3 C . \quad a(t)=e^{x t}
\end{gathered}
$$

From (3.3)

$$
-x \tau=e^{-x t}, \quad a(\tau)=-(x \tau)^{-1}
$$

so, (3.7) reduces to

$$
f_{I}^{\prime \prime}+\left[k^{2}+\frac{1}{x^{2} \tau^{2}}\left(m_{5}+\frac{n M_{c}}{x \tau}\right)^{2}-i \frac{d}{d \tau}\left\{\frac{1}{x \tau}\left(m_{5}+\frac{n M_{c}}{x \tau}\right)\right\}\right] f_{I}=0
$$

Case 1 When $m_{5}>>\frac{n M_{c}}{x \tau}(3.39)$ is approximated to

$$
\tau^{2} f_{I}^{\prime \prime}+\left[k^{2} \tau^{2}+\frac{m_{5}\left(m_{5}+i x\right)}{x^{2}}\right] f_{I}=0
$$

having exact solution

$$
\begin{aligned}
f_{I}= & \exp \left[ \pm i k \tau+\frac{1}{2}\left(1 \pm i \sqrt{4 a^{2}-1}\right) \ln \tau\right]\left[D_{1}{ }_{1} F_{1}\left(\frac{a}{2}, 2 g,-2 g^{\prime} \tau\right)\right. \\
& \left.+D_{2}\left(-2 g^{\prime} \tau\right)^{ \pm i \sqrt{4 a^{2}-1}}{ }_{1} F_{1}\left(1+\frac{a}{2}-2 g, 2-2 g,-2 g^{\prime} \tau\right)\right]
\end{aligned}
$$

where

$$
a=m_{5}\left(m_{5}+i x\right) x^{-2} \quad, \quad g=\frac{1}{2}\left[1 \pm \sqrt{4 a^{2}-1}\right]
$$


and $g^{\prime}=\mp i k$.

Connecting (3.6) and(3.41)

$$
f_{I I}=\frac{i(k . \sigma)}{k^{2}} \exp \left[ \pm i k \tau+\frac{1}{2}\left(1 \pm i \sqrt{4 a^{2}-1}\right) \ln \tau\right]\left[D_{3} X_{1}(\tau)+D_{4} Y_{1}(\tau)\right]
$$

where

$$
X_{1}(\tau)=\left\{\frac{1 \pm \sqrt{4 a^{2}-1}}{2 \tau} \pm i k-\frac{i}{x \tau}\left(\frac{n M_{c}}{x \tau}+m_{5}\right)-\frac{a g^{\prime}}{2 g}\right\}_{1} F_{1}\left(\frac{a}{2}, 2 g,-2 g^{\prime} \tau\right)
$$

and

$$
\begin{aligned}
& Y_{1}(\tau)=\left(-2 g^{\prime} \tau\right)^{ \pm i \sqrt{4 a^{2}-1}}\left[\left\{\frac{1 \pm i \sqrt{4 a^{2}-1}}{2 \tau} \pm i k-\frac{i}{x \tau}\left(\frac{n M_{c}}{x \tau}+m_{5}\right)+\tau^{-1}\right\} \times\right. \\
& \left.{ }_{1} F_{1}\left(1+\frac{a}{2}-2 g, 2-2 g,-2 g^{\prime} \tau\right)-\frac{(2+a-4 g)}{2(1-g)} g^{\prime}{ }_{1} F_{1}\left(\frac{4+a-4 g}{2}, 3-2 g,-2 g^{\prime} \tau\right)\right]
\end{aligned}
$$

So,

$$
\begin{aligned}
\psi_{k 4 I s}^{n}= & (2 \pi)^{-3 / 2} e^{i(k \cdot x)}\left(-\frac{1}{x \tau}\right)^{-9 / 4} \exp \left[ \pm i k \tau+\frac{1}{2}\left(1 \pm i \sqrt{4 a^{2}-1}\right) \ln \tau\right] \times \\
& {\left[D_{1} u_{s} F_{1}\left(\frac{a}{2}, 2 g,-2 g^{\prime} \tau\right)+\right.} \\
& \left.D_{2} \hat{u}_{s}\left(-2 g^{\prime} \tau\right)^{ \pm i \sqrt{4 a^{2}-1}}{ }_{1} F_{1}\left(\frac{2+a-4 g}{2}, 2-2 g,-2 g^{\prime} \tau\right)\right]
\end{aligned}
$$

and

$$
\begin{aligned}
\psi_{k 4 I I s}^{n}= & (2 \pi)^{-3 / 2} e^{i(k \cdot x)}\left(-\frac{1}{x \tau}\right)^{-9 / 4} \exp \left[ \pm i k \tau+\frac{1}{2}\left(1 \pm i \sqrt{4 a^{2}-1}\right) \ln \tau\right] \times \\
& \frac{i(k \cdot \sigma)}{k^{2}}\left[D_{3} u_{s} X_{1}(\tau)+D_{4} \hat{u}_{s} Y_{1}(\tau)\right]
\end{aligned}
$$

Normalization of these solutions yields

$$
\begin{aligned}
& D_{1}=\sqrt{M_{c} x} e^{-i \pi / 2}\left[2 \sqrt{\pi} k\left|{ }_{1} F_{1}\left(\frac{a}{2}, 2 g, \frac{2 g^{\prime}}{x}\right)\right|\right]^{-1} \\
& D_{2}=\sqrt{M_{c} x} e^{-i \pi / 2}\left[2 \sqrt{\pi}\left|{ }_{1} F_{1}\left(\frac{2+a-4 g}{2}, 2-2 g, \frac{2 g^{\prime}}{x}\right)\right|\right]^{-1} \\
& D_{3}=\sqrt{M_{c} x} e^{-i \pi / 2}\left[2 \sqrt{\pi}\left|X_{1}\left(-\frac{1}{x}\right)\right|\right]^{-1}
\end{aligned}
$$

and

$$
D_{4}=k \sqrt{M_{c} x} e^{-i \pi / 2}\left[2 \sqrt{\pi}\left|Y_{1}\left(-\frac{1}{x}\right)\right|\right]^{-1}
$$

Case II When $m_{5} \gtrsim n M_{c} x \tau, \quad$ (3.39) approximates to

$$
\tau^{2} f_{I}^{\prime \prime}+\left[\alpha+\beta \tau+\gamma \tau^{2}\right] f_{I}=0
$$


where $\quad \alpha=m_{5}^{2} x^{-2} \quad, \quad \beta=m_{5}^{2}(2-i x)\left(n M_{c}\right)^{-1}$

and $\gamma=k^{2}+m_{5}^{3}\left(m_{5}-i x\right)\left(n M_{c}\right)^{-2}$

(3.45)yields the solution

$$
\begin{aligned}
f_{I}= & \exp \left[ \pm i \sqrt{\gamma} \tau+\frac{1}{2}(1 \pm i \sqrt{4 \alpha-1}) \ln \tau\right] \times\left[\tilde{D}_{1}{ }_{1} F_{1}\left(\frac{2 j j^{\prime}+\beta}{2 j^{\prime}}, 2 j,-2 j^{\prime} \tau\right)\right. \\
& \left.+\tilde{D}_{2}\left(-2 j^{\prime} \tau\right)^{1-2 j}{ }_{1} F_{1}\left(\frac{2 j^{\prime}-2 j j^{\prime}+\beta}{2 j^{\prime}}, 2-2 j,-2 j^{\prime} \tau\right)\right]
\end{aligned}
$$

where $j=\frac{1}{2}[1 \pm i \sqrt{4 \alpha-1}]$ and $j^{\prime}= \pm i \sqrt{\gamma}$

Connecting (3.6) and (3.46)

$$
f_{I I}=\frac{i(k . \sigma)}{k^{2}} \exp \left[ \pm i \sqrt{\gamma} \tau+\frac{1}{2}(1 \pm i \sqrt{4 \alpha-1}) \ln \tau\right] \times\left[\tilde{D}_{3} X_{2}(\tau)+\tilde{D}_{4} Y_{2}(\tau)\right]
$$

where

$$
\begin{aligned}
& X_{2}(\tau)=\left\{\frac{1}{2 \tau}(1 \mp i \sqrt{4 \alpha-1}) \mp i \sqrt{\gamma}-\frac{i}{x \tau}\left(\frac{n M_{c}}{x \tau}+m_{5}\right)\right\} \\
& { }_{1} F_{1}\left(\frac{2 j j^{\prime}+\beta}{2 j^{\prime}}, 2 j,-2 j^{\prime} \tau\right)-\frac{\left(2 j j^{\prime}+\beta\right)}{2 j}{ }_{1} F_{1}\left(\frac{2 j j^{\prime}+\beta+2 j^{\prime}}{2 j^{\prime}}, 1+2 j,-2 j^{\prime} \tau\right)
\end{aligned}
$$

and

$$
\begin{aligned}
Y_{2}(\tau)= & {\left[\frac{1}{2 \tau}(1 \mp i \sqrt{4 \alpha-1}) \mp i \sqrt{\gamma}-\frac{i}{x \tau}\left(\frac{n M_{c}}{x \tau}+m_{5}\right)\right]\left(-2 j^{\prime} \tau\right)^{1-2 j} \times } \\
& { }_{1} F_{1}\left(\frac{2 j^{\prime}-2 j j^{\prime}+\beta}{2 j^{\prime}}, 2-2 j,-2 j^{\prime} \tau\right)-2 j(1-2 j)(-2 j \tau)^{-2 j} \\
& { }_{1} F_{1}\left(\frac{2 j^{\prime}-2 j j^{\prime}+\beta}{2 j^{\prime}}, 2-2 j,-2 j^{\prime} \tau\right) \\
& -(-2 j \tau)^{1-2 j} \frac{\left(2 j-2 j j^{\prime}+\beta\right)}{2(1-j)}{ }_{1} F_{1}\left(\frac{4 j^{\prime}-2 j j^{\prime}+\beta}{2 j^{\prime}}, 3-2 j,-2 j^{\prime} \tau\right)
\end{aligned}
$$

So

$$
\begin{aligned}
\psi_{k 4 I s}^{n}= & (2 \pi)^{-3 / 2} e^{i(k . x)} \exp \left[ \pm i \sqrt{\gamma} \tau+\frac{1}{2}(1 \pm i \sqrt{4 \alpha-1}) \ln \tau\right] \times \\
& {\left[\tilde{D}_{1} u_{s{ }_{1}} F_{1}\left(\frac{2 j j^{\prime}+\beta}{2 j^{\prime}}, 2 j,-2 j^{\prime} \tau\right)+\right.} \\
& \left.\tilde{D}_{2} \hat{u}_{s}(-2 j \tau)^{1-2 j}{ }_{1} F_{1}\left(\frac{2 j^{\prime}-2 j j^{\prime}+\beta}{2 j^{\prime}}, 2-2 j,-2 j^{\prime} \tau\right)\right]
\end{aligned}
$$

and

$$
\begin{aligned}
\psi_{k 4 I I s}^{n}= & (2 \pi)^{-3 / 2} e^{i k \cdot x} \frac{i(k \cdot \sigma)}{k^{2}} \exp \left[ \pm i \sqrt{\gamma} \tau+\frac{1}{2}(1 \pm i \sqrt{4 \alpha-1}) \ln \tau\right] \times \\
& {\left[\tilde{D}_{3} u_{s} X_{2}(\tau)+\tilde{D}_{4} u_{s} Y_{2}(\tau)\right] }
\end{aligned}
$$


On normalization of these solutions

$$
\begin{aligned}
& \tilde{D}_{1}=\sqrt{M_{c} x} e^{-i \pi / 2}\left[2 k \sqrt{\pi}\left|{ }_{1} F_{1}\left(\frac{2 j j^{\prime}+\beta}{2 j^{\prime}}, 2 j, \frac{2 j^{\prime}}{x}\right)\right|\right]^{-1} \\
& \tilde{D}_{2}=\sqrt{M_{c} x} e^{-i \pi / 2}\left[2 \sqrt{\pi}\left|\left(\frac{2 j}{x}\right)^{1-2 j}{ }_{1} F_{1}\left(\frac{2 j^{\prime}-2 j j^{\prime}+\beta}{2 j^{\prime}}, 2-2 j, \frac{2 j^{\prime}}{x}\right)\right|\right]^{-1} \\
& \tilde{D}_{3}=\sqrt{M_{c} x} e^{-i \pi / 2}\left[2 \sqrt{\pi}\left|X_{2}\left(-\frac{1}{x}\right)\right|\right]^{-1}
\end{aligned}
$$

and

$$
\tilde{D}_{4}=\sqrt{M_{c} x} e^{-i \pi / 2} k\left[2 \sqrt{\pi}\left|Y_{2}\left(-\frac{1}{x}\right)\right|\right]^{-1}
$$

\section{Current for $\psi_{4}^{n}$}

The current is defined as

$$
J_{4}^{\hat{\mu} n}=\bar{\psi}_{4}^{n} \gamma^{\hat{\mu}} \psi_{4}^{n}, \quad(\hat{\mu}=0,1,2,3)
$$

which is divergence - free as $J_{4}^{\hat{\mu} n} ; \hat{\mu}=0$. For a massive field

$$
J_{4}^{\hat{\mu} n}=\bar{\psi}_{4}^{n} \gamma^{\hat{\mu}} \psi_{4}^{n}=\frac{1}{2 M} \bar{\psi}_{4}^{n}\left(i \partial_{\hat{\lambda}} \gamma^{\hat{\lambda}} \gamma^{\hat{\mu}}-i \gamma^{\hat{\mu}} \gamma^{\hat{\lambda}} \partial_{\hat{\lambda}}-i\left[\gamma^{\hat{\lambda}} \Gamma_{\hat{\lambda}}, \gamma^{\hat{\mu}}\right]\right) \psi_{4}^{n}
$$

where $M=m_{5}-a(t) n M_{c}$

which can be re-expressed as

$$
\begin{aligned}
J_{4}^{n \hat{\mu}}= & \frac{1}{2 M}\left(\bar{\psi}_{4}^{n} \sigma^{\hat{\lambda} \hat{\mu}} \psi_{4}^{n}\right), \hat{\lambda}-\frac{i}{4 M} g^{\hat{\mu} \hat{\lambda}} \bar{\psi}_{4}^{n} \overleftrightarrow{\partial_{\hat{\lambda}}} \psi_{4}^{n} \\
& -\frac{i}{4 M} \bar{\psi}_{4}^{n}\left(\left[\gamma^{\hat{\lambda}}, \hat{\lambda}, \gamma^{\hat{\mu}}\right]+\left[\gamma^{\hat{\lambda}}, \gamma^{\hat{\mu}}, \hat{\lambda}\right]\right) \psi_{4}^{n} \\
& -\frac{i}{2 M} \bar{\psi}_{4}^{n}\left[\gamma^{\hat{\lambda}} \Gamma_{\hat{\lambda}}, \gamma^{\hat{\mu}}\right] \psi_{4}^{n}
\end{aligned}
$$

where

$$
\bar{\psi}_{4}^{n} \overleftrightarrow{\partial_{\hat{\lambda}}} \psi_{4}^{n}=\bar{\psi}_{4}^{n} \partial_{\hat{\lambda}} \psi_{4}^{n}-\psi_{4}^{n} \partial_{\hat{\lambda}} \bar{\psi}_{4}^{n}, \quad M=m_{5}-\frac{a(t) n}{k_{c}}
$$

(here $\hat{\mu}, \hat{\nu}, \hat{\lambda}, \ldots$ run from 0 to 3 )

In the $M^{4}$ spacetime

$$
\begin{gathered}
\gamma^{\hat{\lambda}}, \hat{\lambda}=0, \quad\left[\gamma^{\hat{\lambda}}, \gamma^{i}, \hat{\lambda}\right]=\left[\tilde{\gamma}^{0}, \tilde{\gamma}^{i}\right]\left(-\dot{a} \bar{a}^{2}\right) \quad(i=1,2,3) \\
{\left[\tilde{\gamma}^{0}, \gamma^{0},{ }_{0}\right]=0, \quad \sigma^{0 i}=\frac{i}{2 a}\left[\tilde{\gamma}^{0}, \tilde{\gamma}^{i}\right], \quad \sigma^{i j}=\frac{i}{2 a^{2}}\left[\tilde{\gamma}^{i}, \tilde{\gamma}^{j}\right]} \\
\Gamma_{0}=0, \quad \Gamma_{1}=\dot{a} \tilde{\gamma}^{1} \tilde{\gamma}^{0}, \quad \Gamma_{2}=\dot{a} \tilde{\gamma}^{2} \tilde{\gamma}^{0} \text { and } \Gamma_{3}=\dot{a} \tilde{\gamma}^{3} \tilde{\gamma}^{0}
\end{gathered}
$$


So

$$
J_{4}^{n o}=\frac{1}{2 M}\left(\bar{\psi}_{4}^{n} \sigma^{i o} \psi_{4}^{n}\right)_{, i}-\frac{i}{4 M} \bar{\psi}_{4}^{n} \overleftrightarrow{\partial_{0}} \psi_{4}^{n}
$$

and

$$
\begin{aligned}
J_{4}^{n i}= & \frac{1}{2 M} \partial_{0}\left(\bar{\psi}_{4}^{n} \sigma^{o i} \psi_{4}^{n}\right)+\frac{1}{2 M} \partial_{j}\left(\bar{\psi}_{4}^{n} \sigma^{j i} \psi_{4}^{n}\right)+ \\
& \frac{7 i}{2 M}\left(\frac{\dot{a}}{a^{2}}\right) \bar{\psi}_{4}^{n} \tilde{\gamma}^{0} \tilde{\gamma}^{i} \psi_{4}^{n}+\frac{i}{4 M a^{2}} \bar{\psi}_{4}^{n} \overleftrightarrow{\partial_{i}} \psi_{4}^{n}
\end{aligned}
$$

In terms of polarization density and magnetization density $J_{4}^{n o}$ and $J_{4}^{n i}$ is written as

$$
J_{4}^{n o}=\vec{\nabla} \cdot \vec{p}_{4}^{n}+\rho_{4(\text { convective })}^{n}
$$

and

$$
J_{4}^{n i}=\partial_{t} \vec{p}_{4}^{n}+\nabla \times \vec{M}_{4}^{n}+\vec{j}_{4(\text { convective })}^{n}+7\left(\frac{\dot{a}}{a}\right) \vec{p}_{4}^{n}
$$

When $m_{5}>>\frac{a n}{k c}, \quad M \simeq m_{5}$, so $P_{4}^{i n}=\frac{i}{2 m_{5} a} \bar{\psi}_{4}^{n} \tilde{\gamma}^{i} \tilde{\gamma}^{o} \psi_{4}^{n}$

and

$$
\begin{gathered}
M_{4}^{i n}=\epsilon^{i j k}\left(\frac{i}{4 m_{5} a^{2}}\right) \bar{\psi}_{4}^{n}\left[\tilde{\gamma}_{j}, \tilde{\gamma}_{k}\right] \psi_{4}^{n} \\
\rho_{4(\text { convective })}^{n}=-\frac{i}{4 m_{5}} \bar{\psi}_{4}^{n} \overleftrightarrow{\partial_{0}} \psi_{4}^{n}
\end{gathered}
$$

and

$$
J_{4(\text { convective })}^{\text {in }}=-\frac{i}{4 m_{5} a^{4}} \bar{\psi}_{4}^{n} \overleftrightarrow{\partial^{i}} \psi_{4}^{n}
$$

But when $m_{5} \gtrsim \frac{a n}{R_{c}}$, one has

$$
\begin{gathered}
P_{4}^{i n}=\frac{i}{2\left(m_{5}-\frac{a n}{R_{c}}\right) a} \bar{\psi}_{4}^{n} \tilde{\gamma}^{i} \tilde{\gamma}^{o} \psi_{4}^{n} \\
M_{4}^{i n}=\epsilon^{i j k}\left[\frac{i}{4\left(m_{5}-\frac{a n}{R_{c}}\right) a^{2}}\right] \bar{\psi}_{4}^{n}\left[\tilde{\gamma}_{j}, \tilde{\gamma}_{k}\right] \psi_{4}^{n} \\
\rho_{4(\text { convective })}^{n}=-\left[\frac{i}{4\left(m_{5}-\frac{a n}{R_{c}}\right)}\right] \bar{\psi}_{4}^{n} \overleftrightarrow{\partial_{0}} \psi_{4}^{n}
\end{gathered}
$$

and

$$
J_{4(\text { convective })}^{\text {in }}=\frac{i a n}{2 a\left(m_{5} R-a n\right)} \bar{\psi}_{4}^{n} \tilde{\gamma}^{i} \tilde{\gamma}^{o} \psi_{4}^{n}-\frac{i R}{4\left(m_{5} R-a n\right) a^{4}} \bar{\psi}_{4}^{n} \overleftrightarrow{\partial^{i}} \psi_{4}^{n}
$$

Thus, it is found that polarization vector, magnetization density (which is a pseudovector), $\rho_{\text {(convective) }}$ and $J_{(\text {convective) }}[9]$, depend on time. It is interesting to note that when $m_{5} \gtrsim \frac{a n}{R_{c}}$ (which yields realistic fermions ) $J_{(\text {convective) }}$ contains an extra term $\frac{i a n}{2 a\left(m_{5} R-a n\right)} \bar{\psi}_{4}^{n} \tilde{\gamma}^{i} \tilde{\gamma}^{o} \psi_{4}^{n}$. 


\section{References}

[1] Th. Kaluza, Sitzungsber- Preuss. Akad. Wiss. Phys. Math. KI, 966 (1921).

[2] O. Klein, Z. Phys. $\underline{37}$, 895(1926).

[3] B. Dewitt, in Lectures at 1963 Les Houches School, Relativity, Groups and Topology, ed.B. Dewitt and C. Dewitt (Gordon and Breach, New York 1964).

[4] E. Witten, Nucl. Phys. B 186412 (1981).

[5] E. Cremmer and J. Scherk, Nucl. Phys. B 108, 409(1976).

[6] T.Appelquist,A. Chodos and P.G.O. Freund, "Modern Kaluza-Klein Theories" , Addision-Wesley Pub. Comp. Inc. (1987).

[7] A. Davidson, J. Sonnenschein and A. N. Vozmediano, Phys. Rev. D 32, 1330(1985).

[8] A. Chodos and S. Detweiler, Phys. Rev. D 21, 2167(1980).

[9] A.O. Barut and I.H.Duru, Phys. Rev. D $\underline{36}$ 3705(1987) and references therein.

[10] M.J.Duff; in Lectures at the 8th Kyoto Summer Institute Kyoto (1985), Quantum Gravity and Cosmology, ed., H.Sato \& T. Inami, World Scientific Publ.Co. (1986).

[11] J.D. Bjorken and S.D. Drell, Relativistic Quantum Mechanics, McGraw Hill Book Company, 1964.

[12] C.Itzykson and J.B. Zuber, Quantum Field Theory, McGraw-Hill Book Co.

[13] E.A. Lord, Tensors, Relativity and Cosmology, Tata McGraw-Hill Pub. Co. (1976).

[14] T. Appelquist and A. Chodos, Phys. Rev. D $\underline{28}$, 772(1983).

\section{Mustaqeem ANSARI}

Department of Mathematics

North-Eastern Hill University

NEHU Campus, Shillong-793022, India

E-mail: mansari@nehu.ac.in ; mansari12_2006 @yahoo.co.in 